\title{
A New Paranormed Series Space and Matrix Transformations
}

\author{
G. Canan Hazar Güleç*
}

\begin{abstract}
The series space $\left|C_{-1}\right|_{p}$ has been studied for $1 \leq p<\infty$ by Hazar and Sarı̈öl in [9]. The main purpose of this work is to define a new paranormed space $\left|C_{-1}\right|(p)$, where $p=\left(p_{k}\right)$ is a bounded sequence of positive real numbers, which generalizes the results of Hazar and Sarıgöl in [9] to paranormed space. Also, we investigate some topological properties such as the completeness and the isomorphism, and we determine the $\alpha-, \beta-$, and $\gamma$ duals of this paranormed space. Additionally, we give characterization of the classes of infinite matrices $\left(\left|C_{-1}\right|(p), \mu\right)$ and $\left(\mu,\left|C_{-1}\right|(p)\right)$, where $\mu$ is any given sequence space.

Keywords: Paranormed sequence spaces; Absolute summability; Matrix domain; $\alpha-, \beta$ - and $\gamma$ duals; Matrix transformations. AMS Subject Classification (2020): Primary: 40C05 ; Secondary: 40F05; 46A45; 46A35; $46 B 50$.

${ }^{*}$ Corresponding author
\end{abstract}

\section{Introduction}

Any vector subspace of $\omega$, the space of all complex sequences, is called a sequence space. Let $\ell_{\infty}, c$ and $c_{0}$ denote the sets of all bounded, convergent and null sequences, respectively. We write $\ell_{p}=\left\{x=\left(x_{k}\right) \in w: \sum_{k}\left|x_{k}\right|^{p}<\infty\right\}$ for $1 \leq p<\infty$. Also, let $b s$ and $c s$ denote the spaces of all bounded and convergent series, respectively.

A linear topological space $X$ over the real field $\mathbb{R}$ is said to be a paranormed space if there is a subadditive function $g: X \rightarrow \mathbb{R}$ such that $g(\theta)=0, g(x)=g(-x)$ and scalar multiplication is continuous, i.e.,

$$
\left|\alpha_{n}-\alpha\right| \rightarrow 0 \text { and } g\left(x_{n}-x\right) \rightarrow 0 \text { imply } g\left(\alpha_{n} x_{n}-\alpha x\right) \rightarrow 0
$$

for all $\alpha^{\prime} s$ in $\mathbb{R}$ and all $x^{\prime} s$ in $X$, where $\theta$ is the zero vector in the linear space $X$.

Throughout paper, $\left(p_{k}\right)$ is a bounded sequence of strictly positive real numbers such that $H=\sup _{k} p_{k}$ and $M=\max \{1, H\}$. The linear space $\ell(p)$ was defined by Maddox [17, 18] (see also Nakano [22] and Simons [24]) as follows.

$$
\ell(p)=\left\{x=\left(x_{k}\right) \in w: \sum_{k}\left|x_{k}\right|^{p_{k}}<\infty\right\},\left(0<p_{k} \leq H<\infty\right),
$$

which is the complete paranormed space by

$$
g(x)=\left(\sum_{k}\left|x_{k}\right|^{p_{k}}\right)^{1 / M} .
$$

Also, we shall assume throughout that $p_{k}^{-1}+\left(p_{k}^{\prime}\right)^{-1}=1$ provided $1<\inf p_{k} \leq H<\infty$ and we denote the collection of all finite subsets of $\mathbb{N}$ by $\mathcal{F}$. 
Let $X$ and $Y$ be subspaces of $w$ and $A=\left(a_{n v}\right)$ be an arbitrary infinite matrix of complex numbers. By $A x=$ $\left(A_{n}(x)\right)$, we denote the $A$-transform of the sequence $x=\left(x_{v}\right)$, i.e.,

$$
A_{n}(x)=\sum_{v=0}^{\infty} a_{n v} x_{v}
$$

provided that the series is convergent for $n \geq 0$. Then, we say that $A$ defines a matrix transformation from $X$ into $Y$, and denote it by $A \in(X, Y)$ if the sequence $A x=\left(A_{n}(x)\right) \in Y$ for all sequence $x \in X$. By $A_{n}=\left(a_{n \nu}\right)_{\nu=0}^{\infty}$, we denote the sequence in the $n$-th row of $A$.

An infinite matrix $T=\left(t_{n v}\right)$ is called a triangle if $t_{n n} \neq 0$ and $t_{n v}=0$ for all $n, v$ with $v>n[26]$.

For an infinite matrix $A$ and a sequence space $X$, the matrix domain of $A$, which is denoted by $X_{A}$, is defined as

$$
X_{A}=\{x \in w: A x \in X\} .
$$

Several authors have recently defined new paranormed sequence spaces by using matrix domain. For example, Başar and Altay have examined the space $b s(p)$, Altay and Başar have studied the sequence spaces $r^{t}(p), r_{\infty}^{t}(p)$, $r_{c}^{t}(p)$ and $r_{0}^{t}(p)$ in $[4,1,2]$. Also, some new paranormed sequence spaces have been employed by Malkowsky [20], Aydın and Başar [3], Kara and Demiriz [14], Başar et al [5], Yeşilkayagil and Başar [27], Maji and Srivastava [19] and Gökçe and Sarıgöl [8]. Additionally, Malkowsky and Rakočević [21] have obtained some general results on matrix domains of arbitrary triangles which is fundamental for our study.

Moreover, some new sequence and series spaces have been examined by various authors in $[9-13,15,23,28]$. At this point, space $\left|C_{\alpha}\right|_{p}$ for $\alpha>-1$ and $1 \leq p<\infty$, as the set of all series summable by the method $|C, \alpha|_{p}$ defined by Flett in [6], has been defined and studied in [23]. However, for $\alpha=-1$, Thorpe has defined that if the series to sequence transformation

$$
\tau_{n}=\sum_{\nu=0}^{n-1} x_{\nu}+(n+1) x_{n}
$$

tends to a finite number $s$ as $n$ tends to infinity, then the series $\Sigma x_{n}$ is summable by Cesàro summability $(C,-1)$ to the number $s[25]$.

Later on, Hazar and Sarigöl [9] have introduced the space $\left|C_{-1}\right|_{p}$ as the set of all series summable of the method $|C,-1|_{p}$, as follows.

$$
\left|C_{-1}\right|_{p}=\left\{x=\left(x_{n}\right): \sum_{n=1}^{\infty} n^{p-1}\left|\tau_{n}-\tau_{n-1}\right|^{p}<\infty\right\},
$$

where $\left(\tau_{n}\right)$ is defined by $(1.2)$, or

$$
\left|C_{-1}\right|_{p}=\left\{x=\left(x_{n}\right): \sum_{n=1}^{\infty} n^{p-1}\left|(n+1) x_{n}-(n-1) x_{n-1}\right|^{p}<\infty\right\} .
$$

\section{A new paranormed space $\left|C_{-1}\right|(p)$}

In this study, we introduce a new paranormed space $\left|C_{-1}\right|(p)$ by

$$
\left|C_{-1}\right|(p)=\left\{x=\left(x_{n}\right): \sum_{n=1}^{\infty} n^{p_{n}-1}\left|(n+1) x_{n}-(n-1) x_{n-1}\right|^{p_{n}}<\infty\right\},\left(0<p_{n} \leq H<\infty\right) .
$$

If we define the matrix $T(p)=\left(t_{n k}(p)\right)$ by

$$
t_{n k}(p)=\left\{\begin{array}{c}
n^{1 / p_{n}^{\prime}}(n+1), k=n, \\
-n^{1 / p_{n}^{\prime}}(n-1), k=n-1,
\end{array}\right.
$$

then, we can obtain that $x=\left(x_{n}\right) \in\left|C_{-1}\right|(p)$ if and only if $T(p)$-transform of the sequence $x=\left(x_{n}\right)$ is in the space $\ell(p)$. In this way, with the notation of $(1.1)$, we can redefine the space $\left|C_{-1}\right|(p)$ as follows:

$$
\left|C_{-1}\right|(p)=(\ell(p))_{T(p)} .
$$


It is trivial that in the case $p_{n}=p$ for every $n \in \mathbb{N}$, the space $\left|C_{-1}\right|(p)$ is reduced to the space $\left|C_{-1}\right|_{p}, 1 \leq p<\infty$.

Also, we define the sequence $y=\left(y_{n}\right)$ as the $T(p)$-transform of the sequence $x=\left(x_{n}\right)$ by

$$
y_{n}=n^{1 / p_{n}^{\prime}}\left[(n+1) x_{n}-(n-1) x_{n-1}\right] .
$$

In this section, we give some topological results of the newly defined the space $\left|C_{-1}\right|(p)$.

Theorem 2.1. The space $\left|C_{-1}\right|(p)$ is a complete paranormed space with the paranorm defined by

$$
\tilde{g}(x)=\left(\sum_{n}\left|n^{1 / p_{n}^{\prime}}\left[(n+1) x_{n}-(n-1) x_{n-1}\right]\right|^{p_{n}}\right)^{1 / M} .
$$

Proof. Let $x, z \in\left|C_{-1}\right|(p)$. Using Minkowski's inequality, we have

$$
\begin{aligned}
& \left(\sum_{n}\left|n^{1 / p_{n}^{\prime}}\left[(n+1)\left(x_{n}+z_{n}\right)-(n-1)\left(x_{n-1}+z_{n-1}\right)\right]\right|^{p_{n}}\right)^{1 / M} \\
\leq & \left(\sum_{n}\left|n^{1 / p_{n}^{\prime}}\left[(n+1) x_{n}-(n-1) x_{n-1}\right]\right|^{p_{n}}\right)^{1 / M} \\
& +\left(\sum_{n}\left|n^{1 / p_{n}^{\prime}}\left[(n+1) z_{n}-(n-1) z_{n-1}\right]\right|^{p_{n}}\right)^{1 / M} \\
<\infty & \infty
\end{aligned}
$$

Hence, we get $x+z \in\left|C_{-1}\right|(p)$. For any $\alpha \in \mathbb{R}$, since $|\alpha|^{p_{n}} \leq \max \left\{1,|\alpha|^{M}\right\}$, we get $\tilde{g}(\alpha x) \leq \max \{1,|\alpha|\} \tilde{g}(x)$. Thus, $\alpha x \in\left|C_{-1}\right|(p)$. It is obvious that $\tilde{g}(\theta)=0$ and $\tilde{g}(x)=\tilde{g}(-x)$ for all $x \in\left|C_{-1}\right|(p)$ and subadditivity of $\tilde{g}$ is seen from (2.3).

Now take any sequence $\xi=\left(\xi^{n}\right)$, where $\xi^{n}=\left(x_{j}^{n}\right)=\left(x_{0}^{n}, x_{1}^{n}, x_{2}^{n}, \ldots\right) \in\left|C_{-1}\right|(p)$ for each $n \in \mathbb{N}$, such that $\tilde{g}\left(\xi^{n}-x\right) \rightarrow 0$ as $n \rightarrow \infty$ and also, let $\left(\alpha_{n}\right)$ be any sequence of scalars such that $\alpha_{n} \rightarrow \alpha$ as $n \rightarrow \infty$. Then, $\left\{\tilde{g}\left(\xi^{n}\right)\right\}$ is bounded, since the inequality

$$
\tilde{g}\left(\xi^{n}\right) \leq \tilde{g}(x)+\tilde{g}\left(x-\xi^{n}\right) .
$$

So, we have

$$
\begin{aligned}
\tilde{g}\left(\alpha_{k} \xi^{k}-\alpha x\right) & =\left(\sum_{n}\left|n^{1 / p_{n}^{\prime}}\left[(n+1)\left(\alpha_{k} x_{n}^{k}-\alpha x_{n}\right)-(n-1)\left(\alpha_{k} x_{n-1}^{k}-\alpha x_{n-1}\right)\right]\right|^{p_{n}}\right)^{1 / M} \\
& \leq\left|\alpha_{k}-\alpha\right| \tilde{g}\left(\xi^{k}\right)+|\alpha| \tilde{g}\left(\xi^{k}-x\right) \rightarrow 0 \text { as } k \rightarrow \infty .
\end{aligned}
$$

This implies that scalar multiplication is continuous. Hence, $\tilde{g}$ is a paranorm on the space $\left|C_{-1}\right|(p)$.

It remains to prove the completeness of the space $\left|C_{-1}\right|(p)$ with respect to the paranorm $\tilde{g}$. Let $\left(x^{n}\right)$ be any Cauchy sequence in the space $\left|C_{-1}\right|(p)$. Then, for a given $\varepsilon>0$ there exists a positive integer $n_{0}$ such that

$$
\tilde{g}\left(x^{i}-x^{j}\right)<\varepsilon \text { for all } i, j \geq n_{0} .
$$

By definition of $\tilde{g}$ for each fixed $n \in \mathbb{N}$, we have

$$
\left|T_{n}(p)\left(x^{i}\right)-T_{n}(p)\left(x^{j}\right)\right| \leq\left(\sum_{n}\left|T_{n}(p)\left(x^{i}\right)-T_{n}(p)\left(x^{j}\right)\right|^{p_{n}}\right)^{1 / M}<\varepsilon
$$

for all $i, j \geq n_{0}$, which leads us to the fact that the $\left(T_{n}(p)\left(x^{i}\right)\right)$ is a Cauchy sequence of scalars for every fixed $n \in \mathbb{N}$ and hence converges for every $n \in \mathbb{N}$, since $\mathbb{C}$ is complete. So, we write

$$
\lim _{i \rightarrow \infty} T_{n}(p)\left(x^{i}\right)=T_{n}(p)(x) .
$$

Using these infinitely many limits, we may write the sequence $\left\{T_{1}(p)(x), T_{2}(p)(x), \ldots\right\}$. 
We have from (2.4) with $j \rightarrow \infty$,

$$
\left(\sum_{n}\left|T_{n}(p)\left(x^{i}\right)-T_{n}(p)(x)\right|^{p_{n}}\right)^{1 / M}<\varepsilon
$$

for all $i \geq n_{0}$. Thus, $\left(x^{i}\right)$ converges to $x$ in $\left|C_{-1}\right|(p)$.

To show $x \in\left|C_{-1}\right|(p)$, using Minkowski's inequality, we have

$$
\begin{aligned}
& \left(\sum_{n}\left|n^{1 / p_{n}^{\prime}}\left[(n+1) x_{n}-(n-1) x_{n-1}\right]\right|^{p_{n}}\right)^{1 / M} \\
\leq & \left(\sum_{n}\left|n^{1 / p_{n}^{\prime}}\left[(n+1)\left(x_{n}-x_{n}^{i}\right)-(n-1)\left(x_{n-1}-x_{n-1}^{i}\right)\right]\right|^{p_{n}}\right)^{1 / M} \\
& +\left(\sum_{n}\left|n^{1 / p_{n}^{\prime}}\left[(n+1) x_{n}^{i}-(n-1) x_{n-1}^{i}\right]\right|^{p_{n}}\right)^{1 / M} \\
= & \tilde{g}\left(x-x^{i}\right)+\tilde{g}\left(x^{i}\right)<\infty .
\end{aligned}
$$

This shows that $x \in\left|C_{-1}\right|(p)$. Therefore, we have shown that $\left|C_{-1}\right|(p)$ is complete.

Theorem 2.2. The space $\left|C_{-1}\right|(p)$ is linearly isomorphic to the space $\ell(p)$, i.e.,

$$
\left|C_{-1}\right|(p) \cong \ell(p),
$$

where $0<p_{n} \leq H<\infty$ for all $n \in \mathbb{N}$.

Proof. We should show that there exists a bijective linear map from $\left|C_{-1}\right|(p)$ to $\ell(p)$. With $(2.1)$, we define a map

$$
T(p):\left|C_{-1}\right|(p) \rightarrow \ell(p)
$$

by $T(p)(x)=y$, where $y=\left(y_{n}\right)$ is as in $(2.2)$. Then, it is clear that $T(p)$ is linear operator. Also, $T(p)(x)=\theta$ implies $x=\theta$, thus $T(p)$ is injective. Let $y \in \ell(p)$, take the sequence $x=\left(x_{n}\right)$ by

$$
x_{0}=y_{0} \text { and } x_{n}=\sum_{v=1}^{n} \frac{v^{1 / p_{v}}}{n(n+1)} y_{v} .
$$

Then,

$$
\tilde{g}(x)=\left(\sum_{n}\left|T_{n}(p)(x)\right|^{p_{n}}\right)^{1 / M}=\tilde{g}_{1}(y)<\infty,
$$

where $\tilde{g}_{1}$ is the usual paranorm on $\ell(p)$. Thus, we have that $x \in\left|C_{-1}\right|(p)$, and so $T(p)$ is surjective and is paranorm preserving. Hence, $T(p)$ is a linear bijection and the spaces $\left|C_{-1}\right|(p)$ and $\ell(p)$ are linearly isomorphic, which completes the proof.

A sequence $\left(b_{k}\right)$ of the elements of $X$ is called a basis for a sequence space $X$ paranormed by $g$ if and only if, for each $x \in X$, there exists a unique sequence $\left(\lambda_{n}\right)$ of scalars such that

$$
g\left(x-\sum_{k=0}^{n} \lambda_{k} b_{k}\right) \rightarrow 0 \text { as } n \rightarrow \infty
$$

and in this case we write $x=\sum_{k=0}^{\infty} \lambda_{k} b_{k}$.

Since $\left|C_{-1}\right|(p) \cong \ell(p)$, the inverse image of the basis of the space $\ell(p)$ is the basis for our new space $\left|C_{-1}\right|(p)$. So we have the following theorem. 
Theorem 2.3. Let $\mu_{k}=(T(p) x)_{k}$, for all $k \in \mathbb{N}$. Define the sequence $b^{(v)}=\left(b_{n}^{(v)}\right)$ as

$$
b_{n}^{(v)}=\left\{\begin{array}{c}
\frac{v^{1 / p_{v}}}{n(n+1)}, 1 \leq v \leq n \\
0, v>n .
\end{array}\right.
$$

The sequence $b^{(v)}$ is a basis for the space $\left|C_{-1}\right|(p)$ and any $x \in\left|C_{-1}\right|$ (p) has a unique representation of the form

$$
x=\sum_{v=0}^{\infty} \mu_{v} b^{(v)}
$$

\section{Dual spaces and matrix transformations}

In this section, we state and prove the theorems determining the alpha, beta and gamma duals of the space $\left|C_{-1}\right|(p)$ and also characterize the classes of infinite matrices $\left(\left|C_{-1}\right|(p), \mu\right)$ and $\left(\mu,\left|C_{-1}\right|(p)\right)$, where $\mu$ is any given sequence space.

We start with the definition of the $\alpha-, \beta-$, and $\gamma$ duals.

For the sequence spaces $X$ and $Y$ define the set $S(X, Y)$ by

$$
S(X, Y)=\left\{a=\left(a_{k}\right) \in w: x a=\left(x_{k} a_{k}\right) \in Y \text { for all } x \in X\right\} .
$$

Then, the sets

$$
X^{\alpha}=S\left(X, \ell_{1}\right), X^{\beta}=S(X, c s) \text { and } X^{\gamma}=S(X, b s)
$$

are called the $\alpha-, \beta-$ and $\gamma-$ duals of the sequence space $X$, respectively.

Let $B \in\{n \in \mathbb{N}: n \geq 2\}$ and define the sets $E_{1}(p), E_{2}(p), E_{3}(p), E_{4}(p)$ and $E_{5}(p)$ as follows:

$$
\begin{gathered}
E_{1}(p)=\left\{a=\left(a_{k}\right) \in w: \sup _{N \in \mathcal{F}} \sup _{k \in \mathbb{N}}\left|\sum_{n \in N} \frac{k^{1 / p_{k}} a_{n}}{n(n+1)}\right|^{p_{k}}<\infty\right\}, \\
E_{2}(p)=\cup_{B>1}\left\{a=\left(a_{k}\right) \in w: \sup _{N \in \mathcal{F}} \sum_{k}\left|\sum_{n \in N} \frac{k^{1 / p_{k}} a_{n}}{n(n+1)} B^{-1}\right|^{p_{k}^{\prime}}<\infty\right\}, \\
E_{3}(p)=\left\{a=\left(a_{k}\right) \in w: \sup _{n, k \in \mathbb{N}}\left|\sum_{r=k}^{n} \frac{k^{1 / p_{k}} a_{r}}{r(r+1)}\right|^{p_{k}}<\infty\right\}, \\
E_{4}(p)=\cup_{B>1}\left\{a=\left(a_{k}\right) \in w: \sup _{n \in \mathbb{N}} \sum_{k=1}^{n}\left|\sum_{r=k}^{n} \frac{k^{1 / p_{k}} a_{r}}{r(r+1)} B^{-1}\right|^{p_{k}^{\prime}}<\infty\right\}, \\
E_{5}(p)=\left\{a=\left(a_{k}\right) \in w: \sum_{r=k}^{\infty} \frac{k^{1 / p_{k}} a_{r}}{r(r+1)}<\infty, \text { for all } k \in \mathbb{N}\right\} .
\end{gathered}
$$

Lemma 3.1. (see, [7]) (i) Let $1<p_{\nu} \leq H<\infty$ for all $\nu \in \mathbb{N}$. Then, $A \in\left(\ell(p), \ell_{1}\right)$ if and only if there exists an integer $B>1$ such that

$$
\sup _{N \in \mathcal{F}} \sum_{\nu}\left|\sum_{n \in N} a_{n \nu} B^{-1}\right|^{p_{\nu}^{\prime}}<\infty .
$$

(ii) Let $0<p_{\nu} \leq 1$ for all $\nu \in \mathbb{N}$. Then, $A \in\left(\ell(p), \ell_{1}\right)$ if and only if

$$
\sup _{N \in \mathcal{F}} \sup _{\nu \in \mathbb{N}}\left|\sum_{n \in N} a_{n \nu}\right|^{p_{\nu}}<\infty .
$$


We may state the following theorem which computes the $\alpha$-dual of the space $\left|C_{-1}\right|(p)$.

Theorem 3.1. (i) Let $0<p_{\nu} \leq 1$ for all $\nu \in \mathbb{N}$. Then,

$$
\left\{\left|C_{-1}\right|(p)\right\}^{\alpha}=E_{1}(p) .
$$

(ii) Let $1<p_{\nu} \leq H<\infty$ for all $\nu \in \mathbb{N}$.Then,

$$
\left\{\left|C_{-1}\right|(p)\right\}^{\alpha}=E_{2}(p) .
$$

Proof. Since the case (i) may be proved by analogy, we prove only case (ii).

Let $p_{\nu}>1$, take any $a=\left(a_{n}\right) \in w$ and $x \in\left|C_{-1}\right|(p)$. Then, we easily derive that

$$
a_{n} x_{n}=a_{n} \sum_{v=1}^{n} \frac{v^{1 / p_{v}}}{n(n+1)} y_{v}=\sum_{v=1}^{n} \frac{a_{n} v^{1 / p_{v}}}{n(n+1)} y_{v}=\delta_{n}(y), \quad(n \in \mathbb{N})
$$

where $\delta_{n}=\left(\delta_{n v}\right)$ is defined by

$$
\delta_{n v}=\left\{\begin{array}{c}
\frac{a_{n} v^{1 / p_{v}}}{n(n+1)}, 1 \leq v \leq n, \\
0, v>n .
\end{array}\right.
$$

Thus, we can see that $a x=\left(a_{n} x_{n}\right) \in \ell_{1}$ whenever $x \in\left|C_{-1}\right|(p)$ if and only if $\delta y \in \ell_{1}$ whenever $y \in \ell(p)$. This means that $a=\left(a_{n}\right) \in\left\{\left|C_{-1}\right|(p)\right\}^{\alpha}$ if and only if $\delta \in\left(\ell(p), \ell_{1}\right)$. By using Lemma 3.1. (i), we have $\left\{\left|C_{-1}\right|(p)\right\}^{\alpha}=$ $E_{2}(p)$.

Lemma 3.2. (see, [16]) (i) Let $1<p_{\nu} \leq H<\infty$ for all $\nu \in \mathbb{N}$. Then, $A \in\left(\ell(p), \ell_{\infty}\right)$ if and only if there exists an integer $B>1$ such that

$$
\sup _{n \in \mathbb{N}} \sum_{\nu}\left|a_{n \nu} B^{-1}\right|^{p_{\nu}^{\prime}}<\infty
$$

(ii) Let $0<p_{\nu} \leq 1$ for all $\nu \in \mathbb{N}$. Then, $A \in\left(\ell(p), \ell_{\infty}\right)$ if and only if

$$
\sup _{n, \nu \in \mathbb{N}}\left|a_{n \nu}\right|^{p_{\nu}}<\infty
$$

In the following theorem, we characterize the $\gamma-$ dual of the space $\left|C_{-1}\right|(p)$.

Theorem 3.2. (i) Let $0<p_{\nu} \leq 1$ for all $\nu \in \mathbb{N}$. Then,

$$
\left\{\left|C_{-1}\right|(p)\right\}^{\gamma}=E_{3}(p) .
$$

(ii) Let $1<p_{\nu} \leq H<\infty$ for all $\nu \in \mathbb{N}$.Then,

$$
\left\{\left|C_{-1}\right|(p)\right\}^{\gamma}=E_{4}(p) .
$$

Proof. Again, we prove only case (ii). Let $p_{\nu}>1$, take any $a=\left(a_{n}\right) \in w$ and $x \in\left|C_{-1}\right|(p)$. Consider the equation

$$
\sum_{k=1}^{n} a_{k} x_{k}=\sum_{k=1}^{n} a_{k} \sum_{v=1}^{k} \frac{v^{1 / p_{v}}}{k(k+1)} y_{v}=\sum_{v=1}^{n} \sum_{k=v}^{n} \frac{a_{k} v^{1 / p_{v}}}{k(k+1)} y_{v}=\bar{D}_{n}(y)
$$

where $\bar{D}=\left(\bar{d}_{n v}\right)$ is defined by

$$
\bar{d}_{n v}=\left\{\begin{array}{c}
\sum_{k=v}^{n} \frac{a_{k} v^{1 / p_{v}}}{k(k+1)}, 1 \leq v \leq n, \\
0, v>n .
\end{array}\right.
$$

Thus, we deduce from Lemma 3.2 (i) with (3.3) that $a x=\left(a_{k} x_{k}\right) \in b s$ whenever $x \in\left|C_{-1}\right|(p)$ if and only if $\bar{D} y \in \ell_{\infty}$ whenever $y \in \ell(p)$. This means that $a=\left(a_{n}\right) \in\left\{\left|C_{-1}\right|(p)\right\}^{\gamma}$ if and only if $\bar{D} \in\left(\ell(p), \ell_{\infty}\right)$. Therefore, we obtain from Lemma 3.2(i) that $\left\{\left|C_{-1}\right|(p)\right\}^{\gamma}=E_{4}(p)$. 
Lemma 3.3. (see, [16]) Let $0<p_{\nu} \leq H<\infty$ for all $\nu \in \mathbb{N}$. Then, $A \in(\ell(p), c)$ if and only if $(3.1),(3.2)$ hold, and

$$
\lim _{n \rightarrow \infty} a_{n \nu}=\xi_{\nu,}(\nu \in \mathbb{N})
$$

also holds.

Now, we characterize the $\beta$-dual of the space $\left|C_{-1}\right|(p)$.

Theorem 3.3. (i) Let $0<p_{\nu} \leq 1$ for all $\nu \in \mathbb{N}$. Then,

$$
\left\{\left|C_{-1}\right|(p)\right\}^{\beta}=E_{3}(p) \cap E_{5}(p),
$$

(ii) Let $1<p_{\nu} \leq H<\infty$ for all $\nu \in \mathbb{N}$. Then,

$$
\left\{\left|C_{-1}\right|(p)\right\}^{\beta}=E_{4}(p) \cap E_{5}(p) .
$$

Proof. We observe from Lemma 3.3 with (3.3) that $a x=\left(a_{k} x_{k}\right) \in c s$ whenever $x \in\left|C_{-1}\right|(p)$ if and only if $\bar{D} y \in c$ whenever $y \in \ell(p)$. This means that $a=\left(a_{n}\right) \in\left\{\left|C_{-1}\right|(p)\right\}^{\beta}$ if and only if $\bar{D} \in(\ell(p), c)$, where $\bar{D}=\left(\bar{d}_{n v}\right)$ is defined by (3.4). Therefore we derive from Lemma 3.3 that

$$
\sup _{n \in \mathbb{N}} \sum_{k=1}^{n}\left|\sum_{r=k}^{n} \frac{k^{1 / p_{k}} a_{r}}{r(r+1)} B^{-1}\right|^{p_{k}^{\prime}}<\infty \text { and } \lim _{m \rightarrow \infty} \sum_{r=k}^{m} \frac{k^{1 / p_{k}} a_{r}}{r(r+1)}<\infty,
$$

which shows that $\left\{\left|C_{-1}\right|(p)\right\}^{\beta}=E_{4}(p) \cap E_{5}(p)$.

After this step, we give two theorems characterizing the classes of infinite matrices $\left(\left|C_{-1}\right|(p), \mu\right)$ and $\left(\mu,\left|C_{-1}\right|(p)\right)$ where $\mu$ is any given sequence space.

Theorem 3.4. Let $\mu$ be any given sequence space. Then, $A=\left(a_{n k}\right) \in\left(\left|C_{-1}\right|(p), \mu\right)$ if and only if $A_{n} \in\left\{\left|C_{-1}\right|(p)\right\}^{\beta}$ for all $n \in \mathbb{N}$ and $R \in(\ell(p), \mu)$, where $R=\left(r_{n k}\right)$ is defined by

$$
r_{n k}=\sum_{j=k}^{\infty} \frac{a_{n j} k^{1 / p_{k}}}{j(j+1)} .
$$

Proof. We prove this theorem in a way similar to that in Yeşilkayagil and Başar [27]. Assume that $\mu$ is any given sequence space and take into account that the spaces $\left|C_{-1}\right|(p)$ and $\ell(p)$ are linearly isomorphic. Let $A \in\left(\left|C_{-1}\right|(p), \mu\right)$ and $y \in \ell(p)$.

$$
\begin{aligned}
(R T(p))_{n k} & =\sum_{j=k}^{\infty} r_{n j} t_{j k}=r_{n k} t_{k k}+r_{n, k+1} t_{k+1, k} \\
& =\sum_{j=k}^{\infty} \frac{a_{n j} k^{1 / p_{k}}}{j(j+1)} k^{1 / p_{k}^{\prime}}(k+1)-\sum_{j=k+1}^{\infty} \frac{a_{n j}(k+1)^{1 / p_{k+1}}}{j(j+1)}(k+1)^{1 / p_{k+1}^{\prime}} k=a_{n k} .
\end{aligned}
$$

Then, $R T(p)$ exists and $A_{n} \in\left\{\left|C_{-1}\right|(p)\right\}^{\beta}$, which yields that $R_{n} \in\{\ell(p)\}^{\beta}$ for each $n \in \mathbb{N}$. Thus, Ry exists for each $y \in \ell(p)$ and

$$
\begin{aligned}
\sum_{k=1}^{m} r_{n k}^{(m)} y_{k} & =\sum_{k=1}^{m} \sum_{j=k}^{m} \frac{a_{n j} k^{1 / p_{k}}}{j(j+1)}\left(k^{1 / p_{k}^{\prime}}\left[(k+1) x_{k}-(k-1) x_{k-1}\right]\right) \\
& =\sum_{j=1}^{m} \frac{a_{n j}}{j(j+1)} \sum_{k=1}^{j} k\left[(k+1) x_{k}-(k-1) x_{k-1}\right]=\sum_{k=1}^{m} a_{n k} x_{k}
\end{aligned}
$$

where $r_{n k}^{(m)}=\sum_{j=k}^{m} \frac{a_{n j} k^{1 / p_{k}}}{j(j+1)}$ for all $n \in \mathbb{N}$. So, by letting $m \rightarrow \infty$ in the equality (3.5), we have $R y=A x$ and this leads us to $R \in(\ell(p), \mu)$. 
Conversely, let $A_{n} \in\left\{\left|C_{-1}\right|(p)\right\}^{\beta}$ for all $n \in \mathbb{N}, R \in(\ell(p), \mu)$ and $x \in\left|C_{-1}\right|(p)$. Then $A x$ exists. So, we deduce from the equality

$$
\sum_{k=1}^{m} a_{n k} x_{k}=\sum_{k=1}^{m} a_{n k} \sum_{v=1}^{k} \frac{v^{1 / p_{v}}}{k(k+1)} y_{v}=\sum_{k=1}^{m} r_{n k}^{(m)} y_{k}
$$

as $m \rightarrow \infty$ that $A x=R y$ and this gives us that $A \in\left(\left|C_{-1}\right|(p), \mu\right)$. This completes the proof.

Theorem 3.5. Let $\mu$ be any given sequence space. Then, $A \in\left(\mu,\left|C_{-1}\right|(p)\right)$ if and only if $F \in(\mu, \ell(p))$, where $F=\left(f_{n k}\right)$ is defined by

$$
f_{n k}=n^{1 / p_{n}^{\prime}}\left((n+1) a_{n k}-(n-1) a_{n-1, k}\right) .
$$

Proof. Let $z \in \mu$ and consider the following equality

$$
\sum_{k=1}^{m} f_{n k} z_{k}=\sum_{k=1}^{m} n^{1 / p_{n}^{\prime}}\left((n+1) a_{n k}-(n-1) a_{n-1, k}\right) z_{k} .
$$

Then, as $m \rightarrow \infty$ in $(3.6)$ we obtain that $(F z)_{n}=(T(p)(A z))_{n}$. So, one can observe that $A z \in\left|C_{-1}\right|(p)$ whenever $z \in \mu$ if and only if $F z \in \ell(p)$ whenever $z \in \mu$. This step concludes the proof.

\section{References}

[1] Altay, B., Başar, F.: On the paranormed Riesz sequence spaces of non-absolute type. Southeast Asian Bull. Math. 26 (5), 701-715 (2003).

[2] Altay, B., Başar, F.: Some paranormed Riesz sequence spaces of non-absolute type. Southeast Asian Bull. Math. 30 (4), 591-608 (2006).

[3] Aydın, C., Başar, F.: Some generalizations of the sequence space. Iranian Journal of Science and Technology, Transaction A: Science. 30, No.A2 (2006).

[4] Başar, F., Altay, B.: Matrix mappings on the space $b s(p)$ and its $\alpha-, \beta-, \gamma-d u a l s$. Aligarh Bull. Math. 21 (1),79-91 (2002).

[5] Başar, F., Altay, B., Mursaleen, M.: Some generalizations of the space bv of p-bounded variation sequences. Nonlinear Analysis: Theory, Methods \& Applications. 68 (2), 273-287 (2008).

[6] Flett, T.M.: On an extension of absolute summability and some theorems of Littlewood and Paley. Proc. London Math. Soc. 7 113-141 (1957).

[7] Grosse-Erdmann, K.G.: Matrix transformations between the sequence spaces of Maddox. J. Math. Anal. Appl. 180 223-238 (1993).

[8] Gökçe, F., Sarıöl M.A.: A new series space $\left|\bar{N}_{p}^{\theta}\right|(\mu)$ and matrix operators with applications. Kuwait Journal of Science. 45 (4), 1-8 (2018).

[9] Hazar Güleç, G.C., Sarı̈öl M. A.: Compact and Matrix Operators on the Space $|C,-1|_{k}$. J. Comput. Anal. Appl. 25 (6), 1014-1024 (2018).

[10] Hazar, G. C., Sarı̈öl M. A.: Absolute Cesàro series spaces and matrix operators. Acta App. Math. 154, 153-165 (2018).

[11] Hazar Güleç, G. C.: Compact Matrix Operators on Absolute Cesàro Spaces. Numer. Funct. Anal. Optim. 41 (1), 1-15 (2020).

[12] İlkhan, M., Kara, E.E.: A new Banach space defined by Euler totient matrix operator. Operators \& Matrices. 13 (2), 527-544 (2019).

[13] İlkhan, M., Demiriz, S., Kara, E.E.: A new paranormed sequence space defined by Euler totient matrix . Karaelmas Science and Engineering Journal. 9 (2), 277-282 (2019).

[14] Kara, E. E., Demiriz, S.: Some New Paranormed Difference Sequence Spaces Derived by Fibonacci Numbers. Miskolc Mathematical Notes. 16 (2), 907-923 (2015). 
[15] Kara, E. E., İlkhan, M.: Some properties of generalized Fibonacci sequence spaces. Linear and Multilinear Algebra. 64 (11), 2208-2223 (2016).

[16] Lascarides, C.G., Maddox, I.J.: Matrix transformations between some classes of sequences. Proc. Camb. Phil. Soc. 68, 99-104 (1970).

[17] Maddox, I.J.: Paranormed sequence spaces generated by infinite matrices. Proc. Cambridge Philos. Soc. 64, 335-340 (1968).

[18] Maddox, I.J.: Spaces of strongly summable sequences. Quart. J. Math. Oxford 18 (2), 345-355 (1967).

[19] Maji, A., Srivastava, P.: Some Paranormed Difference Sequence Spaces of Order m Derived by Generalized Means and Compact Operators. arXiv:1308.2667v2 (2013).

[20] Malkowsky, E.: Recent results in the theory of matrix transformations in sequence spaces. Mat. Vesnik 49, 187-196 (1997).

[21] Malkowsky, E., Rakočević, V.: On matrix domain of triangles. Appl. Math. Comp. 189 (2), 1146-1163 (2007).

[22] Nakano, H.: Modulared sequence spaces. Proc. Jpn. Acad. 27 (2) 508-512 (1951).

[23] Sarıöl, M.A.: Spaces of series summable by absolute Cesàro and matrix operators. Comm. Math. Appl. 7 (1), 11-22 (2016).

[24] Simons, S.: The sequence spaces $\ell\left(p_{v}\right)$ and $m\left(p_{v}\right)$ Proc. London Math. Soc. 15 (3), 422-436 (1965).

[25] Thorpe, B.: Matrix transformations of Cesàro summable Series. Acta Math. Hung. 48 (3-4), 255-265 (1986).

[26] Wilansky, A.: Summability Through Functional Analysis, North-Holland Mathematical Studies. vol. 85, Elsevier Science Publisher, 1984.

[27] Yeşilkayagil, M., Başar, F.: On the paranormed Nörlund sequence space of nonabsolute type. Abstract and Applied Analysis. Vol. 2014, Article ID: 858704.

[28] Zengin Alp P., İlkhan M.: On the difference sequence space $\ell_{p}\left(\hat{T}^{q}\right)$. Mathematical Sciences and Applications E-Notes. 7 (2), 161-173 (2019).

\section{Affiliations}

G. CANAN HAZAR GÜLEÇ

ADDRESS: Pamukkale University, Dept. of Mathematics, 20070, Denizli-TURKEY.

E-MAIL: gchazar@pau.edu.tr

ORCID ID:0000-0002-8825-5555 